\title{
Inflammatory markers and left ventricular diastolic dysfunction in a family-based population study
}

\author{
Małgorzata Kloch ${ }^{1}$, Katarzyna Stolarz-Skrzypek², Agnieszka Olszanecka², Wiktoria Wojciechowska², \\ Adam Bednarski², Justyna Stefaniak², Danuta Czarnecka² \\ 1Department of Medical Education, Jagiellonian University Medical College, Krakow, Poland \\ $21^{\text {st }}$ Department of Cardiology, Interventional Electrocardiology, and Hypertension, Jagiellonian University Medical College, Krakow, Poland \\ ${ }^{3}$ Department of Bioinformatics and Telemedicine, Jagiellonian University Medical College, Krakow, Poland
}

\begin{abstract}
A b stract
Background: Heart failure affects patients with normal left ventricular systolic function (heart failure with preserved ejection fraction [HFPEF]) and those with reduced ejection fraction (HFREF). The treatment of HFPEF remains an unresolved issue.

Aim: We sought to determinate the relationship between inflammatory markers and left ventricular diastolic dysfunction (LVDD) in a family-based population study.

Methods: A total of 303 participants from the general population (55\% women, median age 49 years and $45 \%$ men, median age 40 years) underwent echocardiography and measurement of serum inflammatory markers: C-reactive protein (CRP), myeloperoxidase (MPO), and interleukin 6 (IL-6).

Results: Serum IL-6 concentration correlated with peak transmitral late diastolic velocity (A) and pulmonary vein systolic-to-diastolic velocity $(S / D)$ ratio $(p<0.01)$. Moreover, a significant correlation between IL- 6 concentration and E/A ratio and early diastolic peak velocities of the mitral annulus displacement ( $\left.E^{\prime}\right)$ was observed. The association of IL- 6 concentration and peak transmitral early diastolic velocities $(E)$ and the $E / E^{\prime}$ ratio $(p<0.05)$ was noted in men. In addition, the CRP concentration was shown to have an effect on $\mathrm{E} / \mathrm{A}$ ratio in women $(\mathrm{p}<0.05)$. A significant correlation between the CRP concentration and $S / D$ ratio was observed both in women $(p<0.01)$ and men $(p<0.05)$. No significant correlation was found between the level of MPO and LVDD parameters. Additionally, only one predictive model was identified; $\mathrm{E}^{\prime}$ was found to be dependent on IL-6, age, and heart rate in men $\left(p<0.001, R^{2}=0.611\right)$.

Conclusions: The above results suggest that inflammation may lead to the onset of LVDD, probably via vascular endothelial dysfunction.
\end{abstract}

Key words: heart failure, inflammatory markers, left ventricular diastolic dysfunction

Kardiol Pol 2019; 77, 1: 33-39

\section{INTRODUCTION}

Heart failure is a disease that affects patients with normal left ventricular systolic function (so-called heart failure with preserved ejection fraction [HFPEF]) and those with reduced ejection fraction (HFREF) [1]. In the presence of heart failure symptoms, the diagnosis of HFPEF requires the occurrence of left ventricular ejection fraction (LVEF) of at least 50\%, elevated levels of natriuretic peptides (B-type natriuretic peptide $>35 \mathrm{pg} / \mathrm{mL}$, and/or N-terminal pro-B-type natriu- retic peptide [NT-proBNP] $>125 \mathrm{pg} / \mathrm{mL}$ ), and at least one additional criterium: relevant structural heart disease or left ventricular diastolic dysfunction (LVDD) [1]. We should bear in mind that LVDD may occur as an isolated disease in over $20 \%$ of the European population [2, 3]. Moreover, LVDD may precede systolic dysfunction, which means that patients with HFREF often also have LVDD of varying severity [1, 4]. Typical symptoms of HFPEF are caused by increased filling pressure resulting from impaired relaxation and disturbed compliance 
of the left ventricle. Numerous clinical trials have demonstrated that pharmacotherapy had a positive effect in reducing morbidity and mortality only in patients with HFREF. The treatment of isolated HFPEF remains an unresolved issue [5].

According to the latest guidelines of the American Heart Association (AHA) and the American College of Cardiology (ACC), HFPEF treatment should focus on hypertension, maintaining sinus rhythm, preventing tachycardia and myocardial ischaemia, and reducing venous pressure $[6,7]$.

Because hypertension affects up to $60 \%$ of patients with HFPEF, the guidelines set by the AHA and ACC place particular emphasis on antihypertensive treatment in such patients $[7,8]$. Normalisation of arterial pressure helps lower left ventricular late diastolic pressure and left atrial pressure, improve left ventricular relaxation, reduce oxygen demand, improve perfusion, and reduce myocardial ischaemia.

The pathogenesis of hypertension is multifactorial and the course of the disease is associated with disturbed interaction between cardiac, vascular, renal, endocrine, genetic, and environmental factors. Experimental and clinical tests suggest that the primary changes in the immune system may have an effect on the inflammatory response of the arterial walls, leading to elevated blood pressure and subsequent hypertension [9-13]. Inflammation is associated with an increase in the serum concentration of many proteins and compounds, such as C-reactive protein (CRP), fibrinogen, interleukin (IL), especially IL-6 and IL-8, and tumour necrosis factor (TNF) [14, 15]. Chronic inflammation leads to endothelial dysfunction $[16,17]$. The endothelium lining of all blood vessels is a key element in regulating vascular resistance [18].

The purpose of this study was to determinate the relationship between inflammatory markers and LVDD in a family-based population study.

\section{METHODS}

\section{Study participants}

The study included participants of the Polish part of a European research project on inherited hypertension called the European Project on Genes in Hypertension (EPOGH). The study was approved by the Ethical Committee of the Jagiellonian University Medical College. All clinical investigations were conducted according to the principles expressed in the Declaration of Helsinki. The study used the family structure and was conducted in the years 1999-2008. The general population families were selected from among the inhabitants of the town and municipality of Niepolomice. In the years 2012-2014, adult members of the previously tested families were re-examined. The subjects were asked to complete a questionnaire providing information on their demographic data, socioeconomic status, comorbidities, as well as the medicines and substances they had taken. The following parameters were measured in each participant: height, body weight, and conventional blood pressure using the average of five consecutive readings obtained during the visit. Hypertension was defined as a blood pressure of at least $140 \mathrm{mmHg}$ systolic or $90 \mathrm{mmHg}$ diastolic (average of five consecutive auscultatory readings at the examination centre). Body mass index was measured as weight in kilograms divided by the square of height in metres. In addition, biochemical markers in serum were taken to determine the levels of NT-proBNP (enzyme immunofluorescence assay), CRP (highly sensitive immunonefelometric assay), myeloperoxidase (MPO), and IL-6 (enzyme-linked immunosorbent assay, R\&D Systems, Minneapolis, MN, USA).

\section{Echocardiography}

All participants refrained from smoking, exercising, and drinking alcohol at least $3 \mathrm{~h}$ before the echocardiography examination.

An experienced physician performed the echocardiography examination using interfaced Vivid7 Pro device (GE Vingmed, Horten, Norway) with a 2.5-3.5-MHz phased-array probe. All the subjects underwent echocardiography according to the standardised protocol, and an experienced examiner analysed the digitally stored image using the EchoPac software version 4.0.4 (GE Vingmed, Horten, Norway). Left ventricular mass was calculated based on the method of Devereux et al. and then adjusted for body surface area to obtain the left ventricular mass index (LVMI) [19]. Based on the transmitral blood flow signal, we measured peak early diastolic velocity $(E)$, peak late diastolic velocity $(A)$, and the early-to-atrial mitral inflow velocity ratio (E/A). On the basis of the tissue Doppler imaging recordings, we measured peak early diastolic mitral annular velocity $\left(E^{\prime}\right)$ and the pulmonary vein systolic-to-diastolic velocity ratio (S/D). We calculated the $E / E^{\prime}$ ratio by dividing transmitral $E$ peak by $E^{\prime}$ velocity.

\section{Statistical analysis}

For continuous variables, the Shapiro-Wilk test was applied as the first step in checking the normality of distribution. Because almost none of the continuous variables had normal distribution, all of them were presented as median and Q1-Q3 quartiles. Categorical variables were shown as a number and percentage of observations. Baseline patient characteristics and differences between women and men were compared using the Mann-Whitney $U$ test and the $\chi^{2}$ test. Spearman's correlation coefficient was used to assess the relationship between the inflammatory and rest continuous parameters. The results were also shown on scatter plots. Additionally, multifactorial models were developed for the logarithmic parameters describing LVDD. Along with the inflammatory parameters, the models took into account other parameters characterising the study group (NT-proBNP level, age, systolic blood pressure, diastolic blood pressure, heart rate, body mass index, treated hypertension, left ventricular internal diameter, ejection fraction, left atrial diameter, and LVMI). 
Table 1. Characteristics of the participants

\begin{tabular}{|c|c|c|c|}
\hline Characteristics & Women $(n=167)$ & Men $(n=136)$ & p \\
\hline \multicolumn{4}{|l|}{ Anthropometric data: } \\
\hline Age [years] & $49.0(36.1-61.4)$ & $40.1(32.2-61.7)$ & 0.170 \\
\hline Age $<50$ years & $80(58.8)$ & $56(41.2)$ & 0.286 \\
\hline Age $\geq 50$ years & $88(52.7)$ & $79(47.3)$ & \\
\hline Height [cm] & $164.0(158.0-168.0)$ & $176.0(172.0-181.0)$ & $<0.001$ \\
\hline Weight $[\mathrm{kg}]$ & $70.0(57.0-79.4)$ & $84.0(74.3-93.4)$ & $<0.001$ \\
\hline Body mass index [kg/m²] & $26.2(21.2-30.1)$ & $26.5(24.4-29.7)$ & 0.056 \\
\hline Waist circumference [cm] & $88.0(74.0-99.0)$ & $96.0(90.0-104.0)$ & $<0.001$ \\
\hline Systolic BP [mmHg] & $122.2(112.1-134.9)$ & $131.2(121.9-140.9)$ & $<0.001$ \\
\hline Diastolic BP [mmHg] & $83.8(76.6-89.3)$ & $85.1(79-92.2)$ & 0.083 \\
\hline Heart rate [bpm] & $74.0(66.0-81.0)$ & $70.5(63.0-78.5)$ & 0.045 \\
\hline \multicolumn{4}{|l|}{ Questionnaire data: } \\
\hline Current smoking & $21(12.6)$ & $23(16.9)$ & 0.287 \\
\hline Alcohol use & $21(12.6)$ & $53(39.0)$ & $<0.001$ \\
\hline Hypertension & $83(49.7)$ & $82(60.3)$ & 0.066 \\
\hline Hypertension treatment & $45(33.1)$ & $60(35.9)$ & 0.605 \\
\hline \multicolumn{4}{|l|}{ Hypertension treatment data: } \\
\hline ACEI or ARB & $21(15.4)$ & $25(15.0)$ & 0.910 \\
\hline$\beta$-blocker & $26(19.1)$ & $41(24.6)$ & 0.257 \\
\hline Diuretic & $21(15.4)$ & $28(16.8)$ & 0.755 \\
\hline Calcium channel blocker & $13(9.6)$ & $24(14.4)$ & 0.203 \\
\hline Aldosterone antagonist & $13(9.6)$ & $12(7.2)$ & 0.455 \\
\hline$\alpha$-blocker & $1(0.7)$ & $0(0.0)$ & 0.499 \\
\hline
\end{tabular}

Values are presented as median (Q1-Q3), or number of subjects (percentage). ACEI — angiotensin converting enzyme inhibitors; ARB — angiotensin receptor blockers; $\mathrm{BP}$ — blood pressure

A p-value $<0.05$ was accepted as statistically significant. All the calculations were carried out using STATISTICA software version 12.0 (StatSoft, Inc., Tulsa, OK, USA).

\section{RESULTS \\ Characteristics of the subjects}

The study involved 303 subjects, including 167 (55\%) women and 136 (45\%) men. The men were significantly taller, had greater body weight and waist circumference, higher systolic blood pressure, greater alcohol intake, and lower heart rate. There was no significant difference in hypertension treatment between women and men (Table 1). The echocardiographic evaluation of the parameters in men revealed greater end-diastolic volume of the left ventricle, thicker interventricular septum and posterior wall of the left ventricle, larger left atrium, greater mass of the left ventricle and lower LVEF. E wave and A wave velocity as well as the $S / D$ ratio were greater in women (Table 2 ). In addition, the analysis of the laboratory tests showed that women had a significantly higher concentration of NT-proBNP and a considerably lower concentration of MPO (Table 3).

The analysis of the influence of inflammatory factors on LVDD parameters demonstrated that the plasma concentra- tion of IL-6 correlated with all the tested parameters of the LVDD in men. Moreover, an increase in IL- 6 was associated with a decrease in the E/A ratio and the $E^{\prime}$ velocity and an increase in the S/D ratio in men and women (Table 4, Fig. 1). Regardless of the sex, a significant positive correlation was observed between S/D and the CRP concentration; in women an additional positive correlation was noted with the $A$ wave and a negative correlation with the E/A ratio. In both women and men, no strong correlation existed between the MPO concentration and any of the parameters of the LVDD. Age significantly correlated with both the inflammatory factors (except MPO) and all LVDD parameters. Detailed results according to age and sex are presented in Table 4. Regarding the multifactorial models, only one model was significant $(p<0.001)$, with $R^{2}=0.611$ for logarithmic parameters of $E^{\prime}$. This model contained IL-6, age, and heart rate and only concerned men (Table 5).

\section{DISCUSSION}

Pharmacotherapy has been proven to reduce morbidity and mortality only in patients with HFREF. Treatment of isolated HFPEF is yet to be determined. Hypertension, the main risk 
Table 2. Echocardiographic characteristics of the participants

\begin{tabular}{|c|c|c|c|}
\hline Characteristics & Women $(n=167)$ & Men $(n=136)$ & $\mathbf{p}$ \\
\hline \multicolumn{4}{|l|}{ Conventional echocardiography: } \\
\hline Left atrial diameter [mm] & $35.0(32.0-39.0)$ & $38.0(36-41)$ & $<0.001$ \\
\hline LV internal diastolic diameter [mm] & $48.0(45.0-51.0)$ & $52.0(50-54)$ & $<0.001$ \\
\hline Interventricular septum thickness [mm] & $9.0(7.0-10.0)$ & $10.0(9-11)$ & $<0.001$ \\
\hline Posterior wall thickness [mm] & $8.0(7.0-10.0)$ & $10.0(8-10)$ & $<0.001$ \\
\hline LV mass index $\left[\mathrm{g} / \mathrm{m}^{2}\right]$ & $78.9(66.6-93.5)$ & $96.1(81.9-106.9)$ & $<0.001$ \\
\hline LV mass $[\mathrm{g}]$ & $138.4(111.0-166.9)$ & $190.7(160.4-221.7)$ & $<0.001$ \\
\hline LV ejection fraction [\%] & $70.0(70.0-77.0)$ & $67.0(62.0-71.0)$ & $<0.001$ \\
\hline \multicolumn{4}{|l|}{ Doppler data: } \\
\hline $\mathrm{E}[\mathrm{cm} / \mathrm{s}]$ & $77.0(66.0-87.5)$ & $73.5(59.0-82.3)$ & 0.033 \\
\hline$A[\mathrm{~cm} / \mathrm{s}]$ & $64.0(54.0-77.0)$ & $57.5(51.0-69.3)$ & 0.003 \\
\hline E/A ratio & $1.18(0.9-1.5)$ & $1.23(1.0-1.5)$ & 0.603 \\
\hline$E^{\prime}[\mathrm{cm} / \mathrm{s}]$ & $11.0(8.0-14.0)$ & $11.0(8.0-14.0)$ & 0.894 \\
\hline $\mathrm{E} / \mathrm{E}^{\prime}$ ratio & $6.80(5.9-8.6)$ & $6.55(5.6-7.8)$ & 0.104 \\
\hline S/D ratio & $1.32(1.13-1.49)$ & $1.20(0.98-1.43)$ & $<0.001$ \\
\hline
\end{tabular}

Values are presented as median (Q1-Q3). A - peak late diastolic velocity; E - peak early diastolic velocity; E' - peak early diastolic mitral annular velocity; E/A — early-to-atrial mitral inflow velocity ratio; E/E' — ratio of early diastolic velocity to early diastolic mitral annular velocity; LV — left ventricular; S/D — pulmonary vein systolic-to-diastolic velocity ratio

Table 3. Biochemical markers in the study population

\begin{tabular}{lccc|} 
Characteristics & Women $(\mathbf{n}=\mathbf{1 6 7})$ & Men $(\mathbf{n}=\mathbf{1 3 6})$ & $\mathbf{p}$ \\
\hline NT-proBNP $[\mathrm{pg} / \mathrm{mL}]$ & $71.5(48.4-113.0)$ & $40.6(21.5-70.7)$ & $<0.001$ \\
CRP $[\mathrm{mg} / \mathrm{L}]$ & $1.38(1.00-2.56)$ & $1.05(1.00-1.79)$ & 0.093 \\
Interleukin 6 [pg/mL] & $1.37(0.99-1.85)$ & $1.30(0.89-1.91)$ & 0.414 \\
Myeloperoxidase $[\mathrm{ng} / \mathrm{mL}]$ & $34.8(22.3-65.1)$ & $52.5(25.5-103.7)$ & $<0.001$ \\
\hline
\end{tabular}

Values are median (Q1-Q3). CRP - C-reactive protein; NT-proBNP - N-terminal pro-B-type natriuretic peptide

factor for heart failure, and its development are associated with the disturbed interaction of many factors, among which the role of inflammatory markers should be emphasised.

The influence of inflammatory factors on LVDD is a relatively new topic in the literature. The results of the first studies to appear on this subject in the last few months have focused on both animal and human populations [20-23].

The present study evaluated the influence of inflammatory factors on the parameters of LVDD in the general population, concentrating on men and women separately. Moreover, correlation between age, inflammatory markers, and LVDD was found; therefore, two age groups were also analysed ( $<50$ and $\geq 50$ years old). It was proven that the plasma concentration of IL- 6 in men weakly correlated with all the tested parameters of LVDD, while in women the only parameters with which it had no significant correlation were peak early diastolic velocity and $E / E^{\prime}$. Additionally, only one LVDD parameter ( $E^{\prime}$ ) for men may be predicted by IL-6, and it is also dependent on age and heart rate. These results suggest that inflammation, which is associated with an increase in the concentration of inflammatory factors, including IL-6, results in vascular endothelial dysfunction, myocardial fibrosis, and consequently, in LVDD.

Fang et al. [20] analysed the association between inflammatory markers and LVDD in patients with hypertrophic cardiomyopathy (HCM). Their study focused on 50 patients with $\mathrm{HCM}$ and a control group of 20 persons. All subjects underwent cardiac magnetic resonance imaging and echocardiography, including an evaluation of LVDD using the $E / A$ wave ratio, mitral annular velocity $\left(E^{\prime}\right)$, and the $E / E^{\prime}$ ratio. In addition, the patients were tested for the concentration of 16 selected inflammatory cytokines. The results showed significantly higher levels of TNF, IL-6, and serum amyloid P component (SAP) in patients with $\mathrm{HCM}$ compared to the control group. Furthermore, the concentrations of IL- 6 and IL-4 and monocyte chemotactic protein (MCP-1) correlated 
Table 4. Relationship between inflammatory markers and left ventricular diastolic dysfunction with and without division into age groups

\begin{tabular}{|c|c|c|c|c|c|c|c|}
\hline & \multicolumn{2}{|c|}{ CRP } & \multicolumn{2}{|c|}{ MPO } & \multicolumn{2}{|c|}{ IL6 } & \multirow[t]{2}{*}{ Age } \\
\hline & Women & Men & Women & Men & Women & Men & \\
\hline \multirow[t]{2}{*}{ Age } & 0.251 ** & & 0.035 & & $0.420 * *$ & & \\
\hline & $0.289 * *$ & $0.204^{*}$ & 0.104 & -0.015 & $0.422^{* *}$ & $0.416^{*}$ & \\
\hline \multirow[t]{3}{*}{$E$} & -0.054 & 0.042 & -0.055 & -0.008 & -0.100 & $-0.175^{*}$ & $-0.492^{* *}$ \\
\hline & $-0.030^{1}$ & $-0.116^{1}$ & $-0.191^{1}$ & $0.012^{1}$ & $-0.229 * 1$ & $-0.210^{1}$ & $-0.486^{\mathrm{M}}$ \\
\hline & $0.016^{2}$ & $-0.034^{2}$ & $-0.038^{2}$ & $-0.213^{2}$ & $0.128^{2}$ & $-0.124^{2}$ & $-0.543^{w}$ \\
\hline \multirow[t]{3}{*}{$A$} & $0.176^{*}$ & 0.156 & 0.091 & 0.038 & $0.240 * *$ & $0.244^{* *}$ & $0.542^{* *}$ \\
\hline & $0.122^{1}$ & $0.389^{* * 1}$ & $-0.054^{1}$ & $0.148^{1}$ & $0.085^{1}$ & $0.195^{1}$ & $0.455^{\mathrm{M}}$ \\
\hline & $0.345^{* * 2}$ & $0.093^{2}$ & $0.094^{2}$ & $0.052^{2}$ & $0.311 * * 2$ & $0.177^{2}$ & $0.586^{w}$ \\
\hline \multirow[t]{3}{*}{$E / A$} & $-0.182^{*}$ & -0.099 & -0.108 & -0.020 & $-0.235^{* *}$ & $-0.235^{* *}$ & $-0.704^{* *}$ \\
\hline & $-0.173^{1}$ & $-0.396^{* * 1}$ & $-0.044^{1}$ & $-0.219^{1}$ & $-0.279^{* * 1}$ & $-0.299 * * 1$ & $-0.619^{\mathrm{M}}$ \\
\hline & $-0.297 * * 2$ & $-0.033^{2}$ & $-0.112^{2}$ & $-0.135^{2}$ & $0.132^{2}$ & $-0.130^{2}$ & $-0.782^{w}$ \\
\hline \multirow[t]{3}{*}{$E^{\prime}$} & -0.112 & -0.87 & -0.066 & 0.038 & $-0.221 * *$ & $-0.395^{* *}$ & $-0.782^{* *}$ \\
\hline & $-0.035^{1}$ & $-0.275^{* * 1}$ & $-0.041^{1}$ & $-0.054^{1}$ & $-0.228^{* * 1}$ & $-0.270 * * 1$ & $-0.720^{\mathrm{M}}$ \\
\hline & $0.005^{2}$ & $-0.438^{* * 2}$ & $-0.056^{2}$ & $-0.014^{2}$ & $-0.139^{2}$ & $-0.360 * * 2$ & $-0.833^{w}$ \\
\hline \multirow[t]{3}{*}{$E / E^{\prime}$} & 0.085 & 0.123 & 0.007 & -0.022 & 0.156 & $0.203^{*}$ & $0.552^{* *}$ \\
\hline & $0.022^{1}$ & $0.083^{1}$ & $-0.074^{1}$ & $0.105^{1}$ & $0.027^{1}$ & $0.027^{1}$ & $0.504^{\mathrm{M}}$ \\
\hline & $0.093^{2}$ & $0.305^{* 2}$ & $0.007^{2}$ & $-0.172^{2}$ & $0.280 * 2$ & $0.193^{2}$ & $0.575^{w}$ \\
\hline \multirow[t]{3}{*}{$S / D$} & $0.286^{* *}$ & $0.200^{*}$ & 0.080 & 0.026 & $0.273^{* *}$ & $0.269^{* *}$ & $0.477^{* *}$ \\
\hline & $0.207^{1}$ & $0.286^{* 1}$ & $0.015^{1}$ & $0.040^{1}$ & $0.220 * 1$ & $0.146^{1}$ & $0.534^{\mathrm{M}}$ \\
\hline & $0.176^{2}$ & $0.003^{2}$ & $0.003^{2}$ & $0.086^{2}$ & $-0.006^{2}$ & $0.179^{2}$ & $0.397 w$ \\
\hline
\end{tabular}

${ }^{*} \mathrm{p}<0.05 ;{ }^{* *} \mathrm{p}<0.01 ; \mathrm{IL}-6$ - interleukin 6; $\mathrm{M}-$ men; $\mathrm{W}-$ women. Others abbreviations - see Tables 2 and 3

Values represent Spearman's correlation coefficients. In the first row analysis without age division.

${ }^{1}$ Analysis for patients aged $<50$ years old; ${ }^{2}$ Analysis for patients aged $\geq 50$ years old

with regional fibrosis, while the levels of stromal cell-derived factor-1 and MCP correlated with diffuse fibrosis. An association existed between fractalkine and interferon $\gamma$ and left ventricular wall thickness. In addition, TNF- $\alpha$, IL-6, SAP, MCP, and IL-10 were associated with LVDD parameters in this study population. It was also shown that the white blood cell count was significantly elevated in patients with $\mathrm{HCM}$, and this was related with diffuse fibrosis and LVDD. Based on these results, it was concluded that the concentration of inflammatory markers was associated with the progression of HCM, and especially with the severity of myocardial fibrosis. In addition, it was found that interference with inflammation might limit the fibrotic process in patients with HCM.

Another experimental study assessed the function of the autonomic and circulatory system, inflammatory markers, and oxidation in two groups of female mice (a group of wild mice [ $n=14]$ and a group of mice with leptin deficiency [ $=14]$ ) [21]. The examined animals underwent an assessment of metabolic parameters, evaluation of heart function using echocardiography, including systolic dysfunction and LVDD (isovolumetric time and the E/A ratio) [22], as well as testing of autonomic and endocrine system indicators and inflammatory and oxidative stress markers. Leptin-deficient animals had increased body weight, as well as higher fasting glucose and triglycerides compared to wild-type mice. Although the left ventricular systolic function was similar in both groups, the leptin-deficient mice were characterised by autonomic dysfunction and LVDD. Moreover, the inflammation markers in this group were higher. On this basis, the researchers speculate that these findings might suggest a relationship between the occurrence of LVDD, diabetes, and obesity in women as well as a significantly higher risk of cardiovascular complications in women compared to men. This may be due to the greater impairment of endothelial response in diabetic women, which is associated with a reduced beneficial oestrogen effect on the cardiovascular system, the promotion of oxidative stress, and inflammation.

Patients with carbohydrate disorders and predictive factors for the development of LVDD were also studied by Mocan et al. [23]. High-sensitivity CRP, fibrinogen, IL-6, IL-8, 

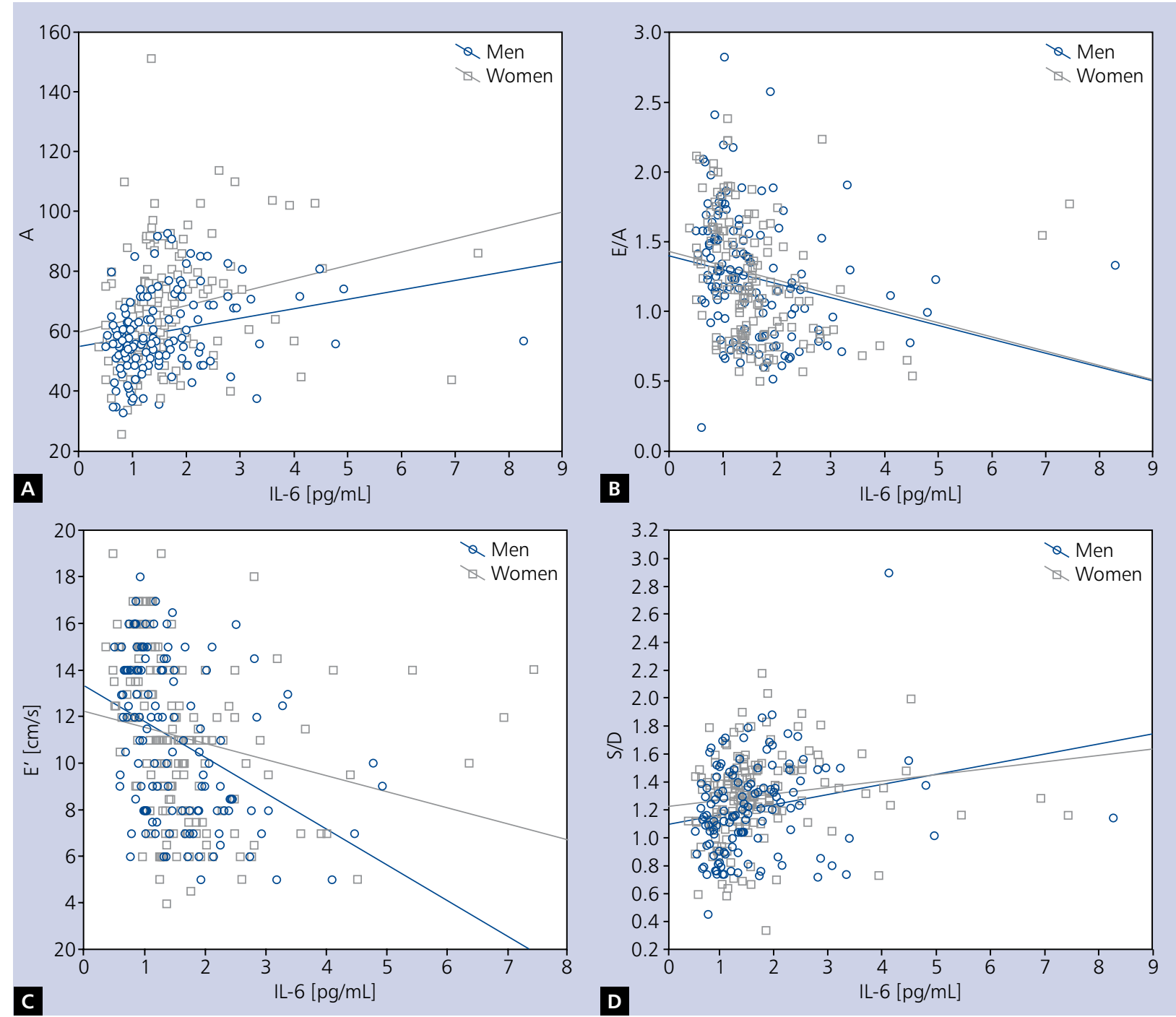

Figure 1. Scatter plots indicating significant correlations between inflammatory markers and left ventricular diastolic dysfunction; IL-6 - interleukin 6; other abbreviations - see Table 2

Table 5. Multifactorial models for the logarithmic parameters of $E^{\prime}$

\begin{tabular}{|lcccc} 
& B & SE(B) & T & p \\
\hline Intercept & 1.518 & 0.060 & 25.360 & $<0.001$ \\
Interleukin 6 & -0.024 & 0.010 & -2.446 & 0.016 \\
Age & -0.006 & 0.001 & -11.887 & $<0.001$ \\
Heart rate & -0.002 & 0.001 & -3.339 & 0.001 \\
\hline
\end{tabular}

$B$ - regression coefficient; $\mathrm{SE}(\mathrm{B})$ - standard error for regression coefficient; $T$ - $t$ statistic test

8-iso-prostaglandin F2 $\alpha$, uric acid, glutathione peroxidase, and NT-proBNP were determined in patients with metabolic syndrome, who had not been previously diagnosed with cardiovascular disease $(n=72)$. This group also underwent echocardiography, and their LVDD was assessed. The tests identified LVDD in approximately $65.27 \%$ of patients. The strongest predictors of LVDD development were diastolic pressure and the number of metabolic syndrome-defining criteria met by the patient.

In addition, IL-6, high-sensitivity CRP, fibrinogen, and NT-proBNP were factors responsible for the development of LVDD. However, it should be emphasised that, similarly to our study, as indicated by multivariate analysis, IL-6 was the only independent prognostic biomarker for LVDD. 
In summary, our study identified in its population a moderate relationship between IL-6 and one of the LVDD parameters $\left(E^{\prime}\right)$ in men. Univariate analysis revealed a correlation of IL-6 and the majority of LVDD parameters; however, after adjustment for age the relationship was not significant.

This observation may suggest a possibility of inflammatory mechanisms related to the development of LVDD. Based on this study it could be speculated that interference with inflammation might limit the development of LVDD. Due to the scarcity of reports on this topic in the literature, further extensive studies are needed to evaluate this relationship.

\section{Conflict of interest: none declared}

\section{References}

1. Ponikowski P, Voors AA, Anker SD, et al. [2016 ESC Guidelines for the diagnosis and treatment of acute and chronic heart failure]. Kardiol Pol. 2016; 74(10): 1037-1147, doi: 10.5603/KP.2016.0141, indexed in Pubmed: 27748494.

2. Kloch-Badelek M, Kuznetsova T, Sakiewicz W, et al. Prevalence of left ventricular diastolic dysfunction in European populations based on cross-validated diagnostic thresholds. Cardiovasc Ultrasound. 2012; 10: 10, doi: 10.1186/1476-7120-10-10, indexed in Pubmed: 22429658.

3. Kloch-Badelek M, Knez J, Tikhonoff V, et al. Heritability and other determinants of left ventricular diastolic function in the family-based population study. J Hypertens. 2014; 32(9): 1854-1861, doi: 10.1097/HJH.0000000000000256, indexed in Pubmed: 25004373.

4. Nagueh SF, Smiseth OA, Appleton CP, et al. Recommendations for the Evaluation of Left Ventricular Diastolic Function by Echocardiography: An Update from the American Society of Echocardiography and the European Association of Cardiovascular Imaging. J Am Soc Echocardiogr. 2016; 29(4): 277-314, doi: 10.1016/j.echo.2016.01.011, indexed in Pubmed: 27037982.

5. Ilieșiu AM, Hodorogea AS. Treatment of Heart Failure with Preserved Ejection Fraction. Adv Exp Med Biol. 2018; 1067: 67-87, doi: 10.1007/5584_2018_149, indexed in Pubmed: 29498023.

6. Yancy CW, Jessup M, Bozkurt B, et al. 2013 ACCF/AHA guideline for the management of heart failure: executive summary: a report of the American College of Cardiology Foundation/American Heart Association Task Force on practice guidelines. Circulation. 2013; 128(16): 1810-1852, doi: 10.1161/CIR.0b013e31829e8807, indexed in Pubmed: 23741057.

7. Yancy CW, Jessup M, Bozkurt B, et al. 2017 ACC/AHA/HFSA Focused Update of the 2013 ACCF/AHA Guideline for the Management of Heart Failure: A Report of the American College of Cardiology/American Heart Association Task Force on Clinical Practice Guidelines and the Heart Failure Society of America. J Card Fail. 2017; 23(8): 628-651, doi: 10.1016/j. cardfail.2017.04.014, indexed in Pubmed: 28461259.

8. Shi Y, Zhou W, Liu X, et al. Resting heart rate and the risk of hypertension and heart failure: a dose-response meta-analysis of prospective studies.
JHypertens. 2018; 36(5): 995-1004, doi: 10.1097/HJH.0000000000001627, indexed in Pubmed: 29176508.

9. Sesso HD, Buring JE, Rifai N, et al. C-reactive protein and the risk of developing hypertension. JAMA. 2003; 290(22): 2945-2951, doi: 10.1001/jama.290.22.2945, indexed in Pubmed: 14665655.

10. Fu ML. Do immune system changes have a role in hypertension? J Hypertens. 1995; 13(11): 1259-1265, indexed in Pubmed: 8984123.

11. Luft FC, Mervaala E, Müller DN, et al. Hypertension-induced end-organ damage: a new transgenic approach to an old problem. Hypertension. 1999; 33(1 Pt 2): 212-218, indexed in Pubmed: 9931107.

12. Nambi V. The use of myeloperoxidase as a risk marker for atherosclerosis. Curr Atheroscler Rep. 2005; 7(2): 127-131, indexed in Pubmed: 15727728.

13. Van der Zwan LP, Scheffer PG, Dekker JM, et al. Hyperglycemia and oxidative stress strengthen the association between myeloperoxidase and blood pressure. Hypertension. 2010; 55(6): 1366-1372, doi: 10.1161/HYPERTENSIONAHA.109.147231, indexed in Pubmed: 20385972.

14. Guzik TJ, Touyz RM. Oxidative Stress, Inflammation, and Vascular Aging in Hypertension. Hypertension. 2017; 70(4): 660-667, doi: 10.1161/HYPERTENSIONAHA.117.07802, indexed in Pubmed: 28784646.

15. Harrison DG, Guzik TJ, Lob HE, et al. Inflammation, immunity, and hypertension. Hypertension. 2011; 57(2): 132-140, doi: 10.1161/HYPERTENSIONAHA.110.163576, indexed in Pubmed: 21149826.

16. Wannamethee SG, Shaper AG, Lowe GDO, et al. Renal function and cardiovascular mortality in elderly men: the role of inflammatory, procoagulant, and endothelial biomarkers. Eur Heart J. 2006; 27(24): 2975-2981, doi: 10.1093/eurheartj/ehl402, indexed in Pubmed: 17132648.

17. Kobayasi R, Akamine EH, Davel AP, et al. Oxidative stress and inflammatory mediators contribute to endothelial dysfunction in high-fat diet-induced obesity in mice. J Hypertens. 2010; 28(10): 2111-2119, doi: 10.1097/HJH.0b013e32833ca68c, indexed in Pubmed: 20616756.

18. Park C, Kim HY, Park HJ, et al. Association between the JNC 7 Classification of the Stages of Systolic Hypertension and Inflammatory Cardiovascular Risk Factors. Korean Circ J. 2007; 37(12): 623, doi: 10.4070/kcj.2007.37.12.623.

19. Schiller NB, Shah PM, Crawford M, et al. Recommendations for quantitation of the left ventricle by two-dimensional echocardiography. American Society of Echocardiography Committee on Standards, Subcommittee on Quantitation of Two-Dimensional Echocardiograms. J Am Soc Echocardiogr. 1989; 2(5): 358-367, indexed in Pubmed: 2698218.

20. Fang Lu, Ellims AH, Beale AL, et al. Systemic inflammation is associated with myocardial fibrosis, diastolic dysfunction, and cardiac hypertrophy in patients with hypertrophic cardiomyopathy. Am J Transl Res. 2017; 9(11): 5063-5073, indexed in Pubmed: 29218105.

21. Sartori M, Conti F, Dias D, et al. Association between diastolic dysfunction with inflammation and oxidative stress in females ob/ob mice. Front Physiol. 2017; 8, doi: 10.3389/fphys.2017.00572.

22. Wichi R, Malfitano C, Rosa K, et al. Noninvasive and invasive evaluation of cardiac dysfunction in experimental diabetes in rodents. Cardiovasc Diabetol. 2007; 6: 14, doi: 10.1186/1475-2840-6-14, indexed in Pubmed: 17462095

23. Mocan M, Anton F, Suciu S, et al. Multimarker Assessment of Diastolic Dysfunction in Metabolic Syndrome Patients. Metab Syndr Relat Disord. 2017; 15(10): 507-514, doi: 10.1089/met.2017.0060, indexed in Pubmed: 29099655.

Cite this article as: Kloch M, Stolarz-Skrzypek K, Olszanecka A, et al. Inflammatory markers and left ventricular diastolic dysfunction in a family-based population study. Kardiol Pol. 2019; 77(1): 33-39, doi: 10.5603/KP.a2018.0214.

\section{WHAT IS NEW?}

Pharmacotherapy has been proven to reduce morbidity and mortality only in patients with heart failure with reduced ejection fraction. Treatment of isolated heart failure with preserved ejection fraction remains undetermined. Hypertension, the main risk factor for heart failure, and its development are associated with the disturbed interaction of many factors, among which the role of inflammatory markers should be emphasised. The results of our study suggest that inflammation, which is associated with an increase in inflammatory markers, including interleukin 6, leads to the onset of left ventricular diastolic dysfunction (LVDD), probably via vascular endothelial dysfunction. Moreover, the study identified in its population a relationship between the concentration of interleukin 6 and the majority of LVDD parameters. This observation may shed new light on the mechanisms related to the development of LVDD. This relationship requires further research, which could change our current views on the treatment of heart failure with preserved ejection fraction. 